\title{
Fully printed microwave sensor for simultaneous and independent level measurements of 8 liquids
}

\author{
Muhammad Akram Karimi ${ }^{1}$, Muhammad Arsalan ${ }^{2}$, Atif Shamim ${ }^{1}$ \\ ${ }^{1}$ IMPACT Lab, Electrical Engineering Dept. King Abdullah University of Science and Technology, Thuwal, KSA \\ ${ }^{2}$ EXPEC Advanced Research Centre, Dhahran, Saudi Aramco, KSA
}

\begin{abstract}
Level sensors find numerous applications in many industries to automate the processes involving chemicals. Currently, some commercial ultrasound, capacitance and microwave radar based level sensors are being used for medical and industrial usage [1]. Some of the desirable features in any level sensor are its non-intrusiveness, low cost and consistent performance. It is a common stereotype to consider microwaves sensing mechanism as being expensive. Unlike usual expensive, intrusive and bulky microwave methods of level sensing using guided radars, this paper presents an extremely low cost, fully printed, non-intrusive microwave sensor to reliably sense the level/volume of 8 different types of liquid independently and simultaneously. This paper presents a new microwave level sensor whose design is inspired by a T-resonator. A unique modification of the conventional T-resonator enables it to sense liquid level/volume present inside any metallic container of cylindrical shape. The proposed sensor can be thoroughly fabricated using additive manufacturing like 3D and screen printing making it easier, faster and cheaper to realize.

Index Terms-Level Sensor, Microwave resonator, T-resonator, 3D printing, Low-cost sensor
\end{abstract}

\section{INTRODUCTION}

Wide range of industrial applications require to store different liquids in storage tanks made of metal. It's crucial to sense the real time liquid level/volume for industrial process automation. An ideal level sensor must be able to provide accurate reading with sufficient resolution and should preferably be low cost. Wide range of sensors based upon the principles of ultrasounds, capacitance and guided microwaves are currently available to do the job. However, ultrasound based level sensors are known to get affected by the surface foams, capacitive sensors have bad performance in the presence of lossy mediums (such as saline water) and microwave radar based sensors are not only expensive but are intrusive as well [2].

Unlike guided radars, researchers have also shown some nonintrusive microwave resonator based sensors to measure the volumetric fraction of different liquids [3], however, these designs can't be used with metallic pipes which is mostly the case in industrial settings.

To the best of authors' knowledge, this paper is presenting a completely new microwave sensor design which can measure the level/volume of 8 different liquids simultaneously and independently. Although, the presented design can easily be machined but we have shown in the paper that it can also be realized using additive manufacturing techniques like $3 \mathrm{D}$ and screen printing in a very fast and cheap manner.

\section{DESIGN CONCEPT \& SimULATIONS}

The presented sensor design is inspired by a microwave Tresonator which is conventionally used to characterize the dielectric properties of the flat and non-changing dielectric substrates. Although, researchers have demonstrated the use of Tresonator to distinguish between different liquids passing through a microfluidic channel [4] but its use is limited to flat and non-metallic surfaces [5]. When it comes to sensing large quantities of liquids in industrial environment with metallic cylinders, guided radar or cavity resonator based microwave sensors are the few expensive options available for now.

In this paper, we are proposing a unique modification of a typical T-resonator to make it compatible to work with metallic cylinders. Typical T-resonator consists of a series feedline with a shunt stub on one side of the substrate, while the ground plane lies on the other side. In our design (shown in Figure 1), we have taken a central dielectric core on which are placed the feedline and the $\lambda / 4$ shunt stub. For matching the microstrip feedline to $50 \Omega$, a dedicated ring shaped ground plane has been provided just underneath the feedline which slightly extends backwards. Feedline and ring ground are separated by a dielectric piece of $1 \mathrm{~mm}$ thickness (white color in Figure 1). The backward extension of the ground plane is shorted with the external metallic enclosure using a shorting rod. With this setting, external cylinder acts as the ground plane for the $\lambda / 4$ shunt stub. Presence of any dielectric medium in between the central core and external metallic cylinder will change the guided wavelength and hence the resonance frequency of the resonator.

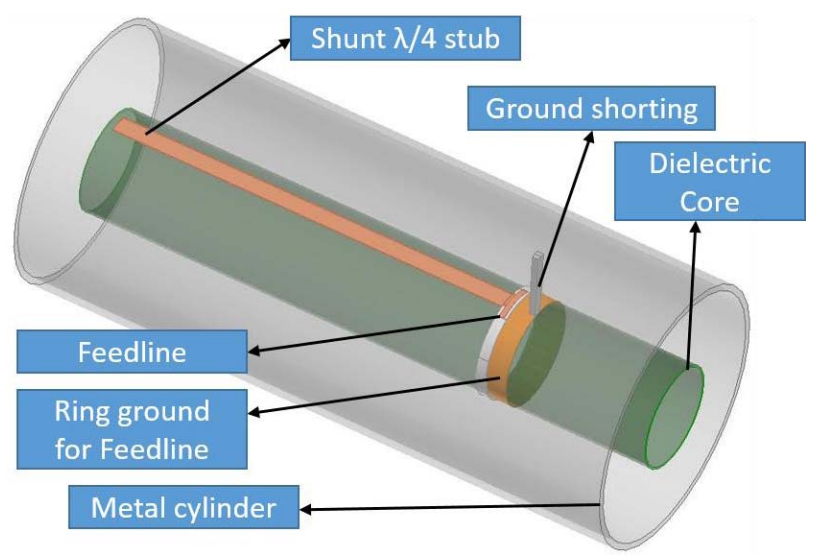

Figure 1. Stage 1 of the design of a modified T-resonator based level sensor 
If we look at the E field distribution in the cross section of the proposed design (shown in Figure 2), we can see that most of the fields are concentrated in $45^{\circ}$ wedge region between central core and external metallic cylinder. From this, we can say that the field of view of a single resonator is around $45^{\circ}$ and up to 8 similar resonators can be packed on the core to cover $360^{\circ}$ field of view (sensing). An interesting observation is that the external metallic cylinder can act as a common ground plane for all the T-resonators. However, there is a tendency for the E-fields of a single resonator to fringe to neighboring region.

As a result, the resonance frequency of a single resonator will be mainly dependent upon the dielectric properties of the medium inside its $45^{\circ}$ region but it will also be slightly affected by the dielectric medium in the neighboring regions.

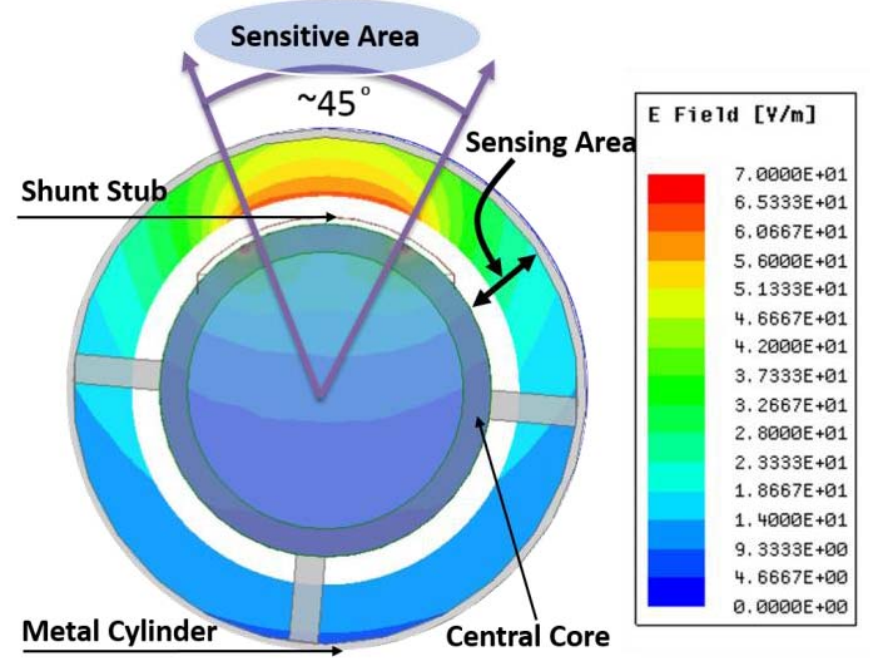

Figure 2. Cross sectional view of the E field distribution in between central core and external metallic cylinder

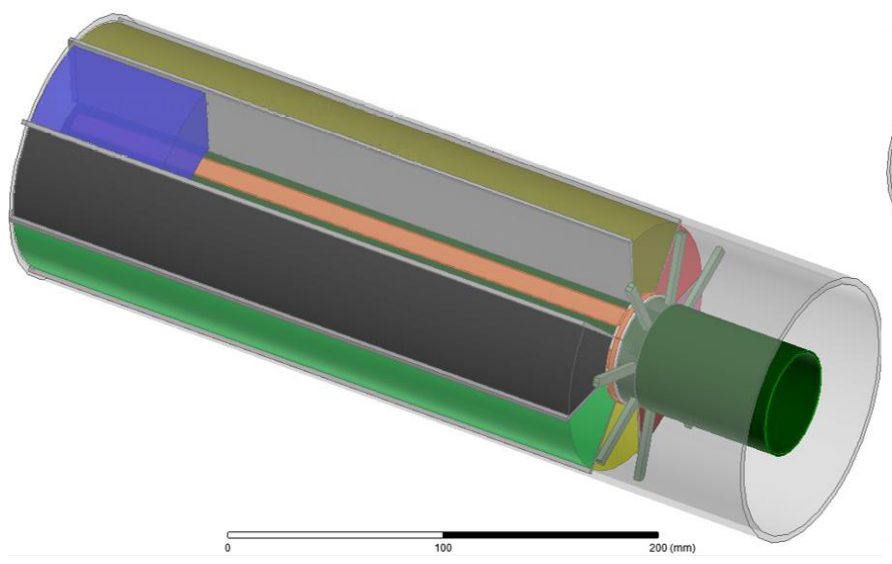

(a) apart and dividing the cylindrical space into 8 sections. These sheets are connected to the external cylindrical ground plane (as shown in Figure 3). With the presence of 2 sheets on either side of the resonator; E fields of a particular resonator will be restricted to its sector only and the resonance frequency of each resonator will be dictated by the dielectric medium in that particular sector only. In our case, these sectors are filled with liquids. In an empty sector, air is filled inside a sector but as the liquid is poured inside a particular sector, effective dielectric properties of the sector changes. It changes the effective wavelength ${ }^{1}$ of the T-resonator which effectively changes its resonance frequency. With this design configuration, we can sense the liquid level/volume of up to 8 liquids simultaneously and independently.

The final design of the proposed sensor is shown in Figure 3 in which 2 " internal core has been used inside a 4 " external metallic enclosure.

\section{FABRICATION}

The simplicity of the proposed design allows it to be realized using low cost and rapid prototyping methods of 3D printing and manual screen printing. All the parts of the sensor including external enclosure, 8 containers to hold liquids, sector isolating sheets and feedline dielectric has been printed using connex 3 object $2603 \mathrm{D}$ printer. The printed parts are shown in Figure 4(a).

PE819 conductive paste from DuPont was used to completely metallize the outer surface of the cylindrical enclosure and sector isolating sheets. These sheets were diagonally inserted inside the cylinder from the spacing kept in the walls of the cylinder which can be seen in Figure 4(a).

Figure 3. (a) Proposed microwave sensor for simultaneous sensing of 8 liquid columns (b) Breakdown of the design

$$
{ }^{1}\left(\lambda_{g}=\lambda_{o} / \sqrt{\epsilon}_{e f f}\right)
$$




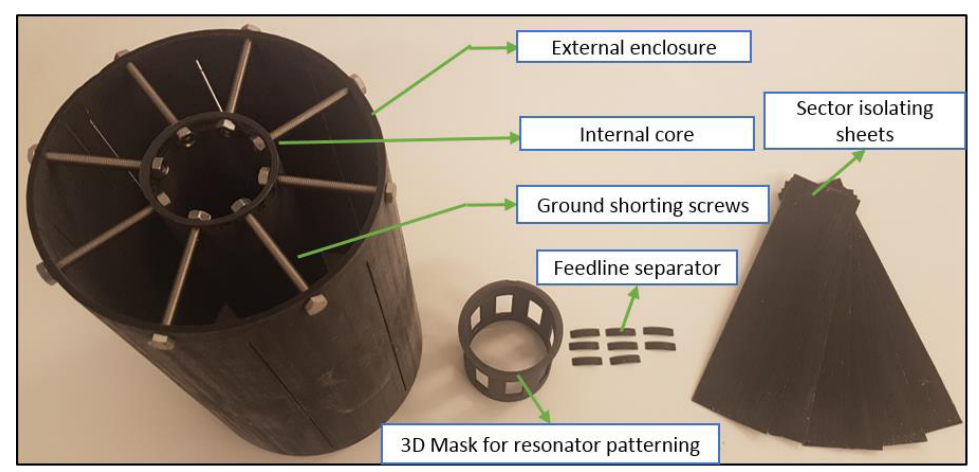

(a)

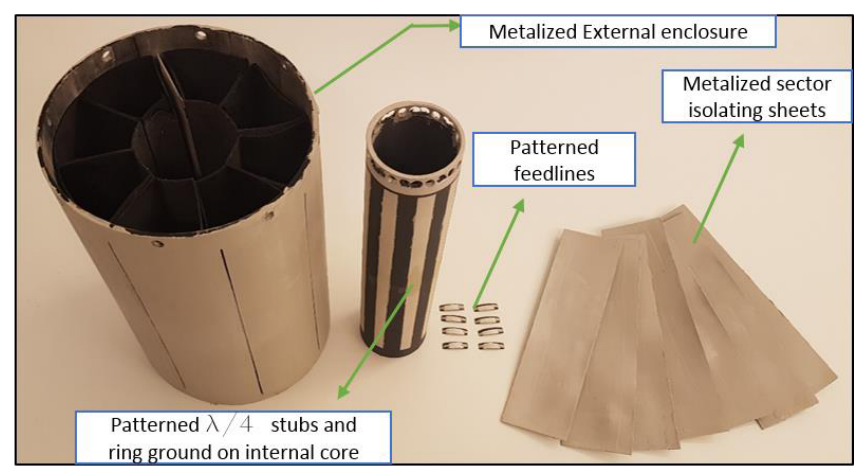

(b)

Figure 4. (a) 3D printed parts of the microwave multi-level sensor (b) Metalized and patterned parts of the sensor using manual screen printing

The resonator fabrication was a bit trickier as it had to be patterned on 3D-printed central core. In order to mutually align 8 shunt stubs on the central core, a 3D printed mask (shown in Figure 4(a)) was prepared and was put on top of central core. The mask had mutually aligned openings for the 8 stubs which helped us to make marking on the core using which 8 perfectly aligned shunt stubs were patterned on the central core as shown in Figure 4(b). Similarly 8 feedlines for these shunt stubs were also patterned on top of small 3D printed dielectric pieces and

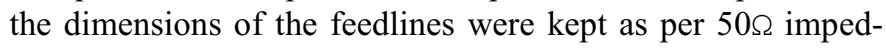
ance $^{2}$ calculations. All the metalized parts are shown in Figure 4(b).

Due to the restricted space, SMA connectors can't directly fit in the prototype so we decided to put the U.FL connectors with the resonators. After placing the connectors with conductive epoxy, 5 minute Devcon epoxy was used to give it more strength. The resonators had to be characterized using a VNA with SMA connectors so an adapter from U.FL to SMA connector was required. Instead of using 16 such adapters ( 2 ports for each resonator), which may also not fit in the system, we have designed a 16 port U.FL to SMA adapter as shown in Figure 5. 16 U.FL female connectors are soldered with 16 edge mounted SMA connectors on a 2" diameter PCB which exactly fit on top of internal core. Using this approach, it became quite easy to route thin U.FL wires out of the resonators to put on the adapter which can then be connected to VNA using SMA connector.

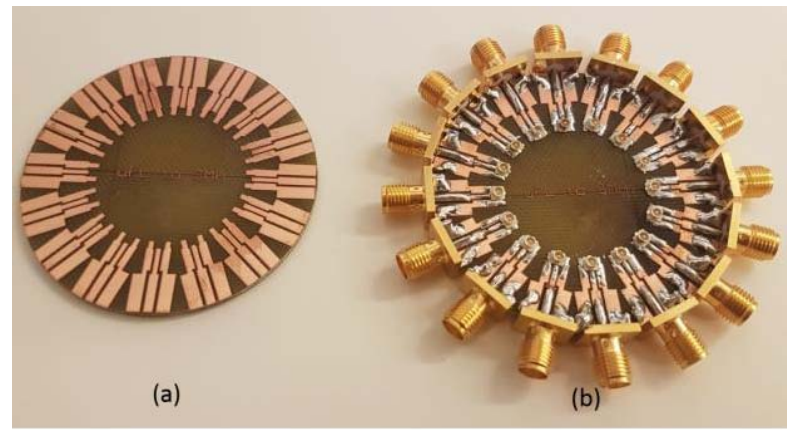

Figure 5. (a) PCB for 16 port U.FL to SMA adapter (b) U.FL to SMA adapter

${ }^{2}$ width of $2.55 \mathrm{~mm}$ on a 3D printed substrate with $\epsilon_{\mathrm{r}}=2.8$ and $\tan \delta=0.02$

\section{MEASUREMENTS}

In order to validate the new sensor design, two set of measurements have been performed on the fabricated system. The measurement system is shown in Figure 6 in which compact $\mathrm{uFL}$ connectors on the resonators are connected to SMA adapter (placed on top of the sensor) which is then connected to the Keysight Fieldfox VNA. In the first set of measurements, one out of 8 sensors have been tested by pouring different water volumes in its respective sector. Its measured transmission parameter $\left(\mathrm{S}_{21}\right)$ can be seen in Figure 7. The sensor has band-stop response like any typical T-resonator. The figure shows that the resonance frequency of the resonator decreases with increase in water volume (up to $174 \mathrm{~mL}$ ) in the sector associated with the sensor under test. As explained earlier that the resonance frequency is inversely proportional to the square root of the effective medium faced by the resonator which in this case is the combination of water and air. As the water has higher dielectric constant, we see a decrease in resonance frequency with its rising content/volume. The resonance frequency changes from $222 \mathrm{MHz}$ to $203 \mathrm{MHz}$ while changing the liquid volumes from $43 \mathrm{~mL}$ to $174 \mathrm{~mL}$. It shows that the presented sensor has a sensitivity of $145 \mathrm{kHz} / \mathrm{mL}$. Although this sensitivity is sufficient to achieve a resolution of $1 \mathrm{~mL}$ or even less, however, the sensitivity depends upon the spacing between the inner dielectric core and outer metallic cylinder. More the space (or effectively more the varying dielectric liquid medium) in between two concentrically placed cylinders, more the sensitivity can be achieved.

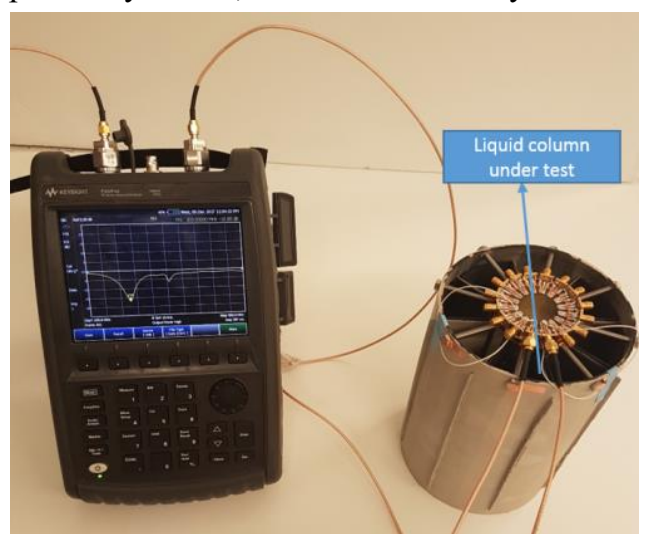

Figure 6. Measurement setup of the sensor 


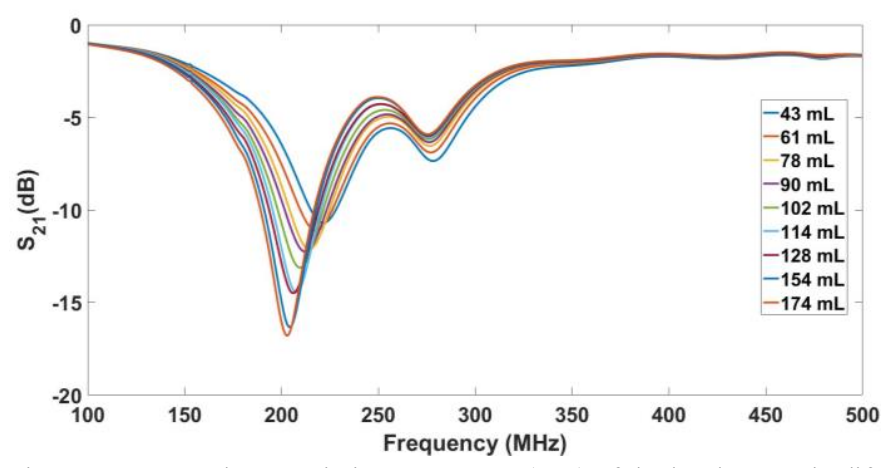

Figure 7. Measured Transmission parameters (S21) of the level sensor in different water volumes

The other set of measurements has been performed to confirm the liquid level measurements in a particular sector is not affected by the liquid content in its neighboring sectors.

To verify this, we measured the resonance frequency of previously tested sensor (or resonator) having water volume of $174 \mathrm{~mL}$ and by pouring different volumes of water (up to $150 \mathrm{~mL}$ ) in its neighboring sectors. As can be seen from Figure that the response of a particular liquid sector is almost independent of liquid volumes present in its neighborhood.

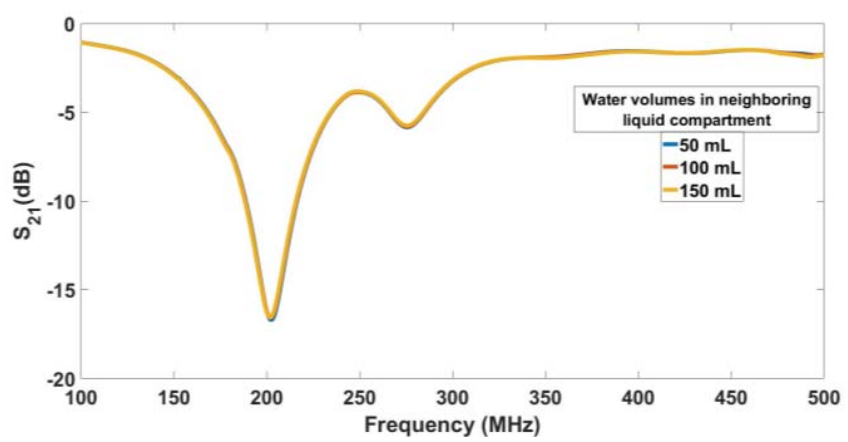

Figure 8. Effect on resonance frequency due to liquid in neighboring compartment of the sensor

With the initial measured results presented in the paper, we can claim that the new type of low-cost and fully printed sensor can sense level/volume of multiple (up to 8) liquids simultaneously and independently.

This paper is mainly focused on characterization of the sensor. Before using this design as a sensor for different liquids, it needs to be calibrated first. Due to its unconventional design, empirical relations of standard T-resonator may not be applicable on this. One way to perform its calibration is to look at the response of the sensor (shift in resonance frequency with different liquid levels) for different known liquids having known dielectric properties to form a database. Curve fitting techniques can then be used to associate the sensor response with the dielectric constant and a relationship can be developed between the dielectric constant (or liquid type) and the characteristic curve of the sensor.

Before testing, sensor response can be measured at two or three known liquid levels which will act as its calibration points for further readings.

\section{CONCLUSION}

The paper has successfully demonstrated a new type of microwave based sensor to measure liquid level/volume in multiple compartments simultaneously and independently. The simplicity of the design has enabled its low cost and rapid prototyping using $3 \mathrm{D}$ and screen printing techniques. Liquid level sensing has been demonstrated successfully in measurements without having any dependence from neighboring liquid sectors. It makes this deign quite suitable for detection of multiliquids simultaneously in industrial environment. In future, a microwave oscillator can be integrated with the resonator to form a self-contained sensor for industrial setting.

\section{REFERENCES}

[1] INTROTEK, "Drip Chamber Ultrasonic Liquid Level Sensors," [Online]. Available: http://introtek.com/productslist/drip-chamber-sensors/drip-chamber/.

[2] VEGA. [Online]. Available: https://www.vega.com/en/home_sv/Company/Blog/2017/Level-comparison-80-GHz-radar-versus-ultrasonic.

[3] M. A. Karimi, M. Arsalan and A. Shamim, "Design and Dynamic Characterization of an Orientation Insensitive Microwave Water-Cut Sensor," IEEE Transactions on Microwave Theory and Techniques, vol. PP, no. 99 , pp. 1 - 10, 2017.

[4] D. C. B. C. A. S. G Mckerricher, "Crude oil water-cut sensing with disposable laser ablated and inkjet printed RF microfluidics," in IMS, 2014.

[5] M. A. Karimi, M. Arsalan and A. Shamim, "Low Cost and Pipe Conformable Microwave-Based Water-Cut Sensor," IEEE Sensors Journal, vol. 16, no. 21, pp. 7636 - 7645, 2016. 